\title{
A CREDIBILITY APPROACH TO MORTALITY RISK
}

\author{
BY \\ M.R. HARdy AND H.H. PANJeR \\ University of Waterloo, Ontario
}

\begin{abstract}
Bühlmann-Straub credibility is used to find an estimate of the mortality loss ratio for a company, relative to a standard table, for use in the statutory valuation of life insurance business. A method for calculating the margin for adverse deviation to be added to the mortality rate (in accordance with the general principle of Canadian statutory valuation) is derived. Applying credibility further to the variance of the mortality loss ratio gives a methodology for calculating the amount of the surplus (i.e. capital) required to cover annual fluctuations in mortality experience. The necessary structural parameters are calculated from industry statistics; the methodology is illustrated using Canadian life insurance data.
\end{abstract}

\section{KEYWORDS}

Solvency; Bühlmann-Straub Credibility; mortality.

\section{INTRODUCTION}

In life insurance practice in most countries, companies compute policy reserves using assumptions which incorporate some safety margins, and, in addition, develop some measure of surplus to be allocated to a provision against unexpected adverse results.

In general, the level at which the valuation margins and additional reserves are imposed tends to be arbitrary, based perhaps on 'actuarial judgement'.

In this paper, we address the risk to solvency from adverse mortality experience for insurance business, though the principles apply equally well for annuity business. We are particularly interested in a methodology that is consistent with the current Canadian valuation requirements, and that could therefore be easily adapted for implementation in Canada. 
Current Canadian regulations require explicit statement of:

1. The 'best estimate' of a company's future mortality experience, assuming no major changes in the nature of the business written;

2. The safety margin to be added to (or subtracted from, where appropriate) the best estimate to give a reserving mortality basis which reflects the uncertainty in the best estimate. The margin plus the best estimate should be greater than the true (unknown) mortality rate with high (currently unstated) probability;

3. A measure of the equalisation or fluctuation reserve (in fact an allocation of surplus) that should be held in addition to the policy reserves, to cushion the company against year-to-year fluctuations in the company mortality experience.

The primary objective of this work therefore is to provide a theoretical foundation for calculating these three quantities, subject to the important constraints that any system developed must be capable of implementation with the data currently available, and without undue expense or difficulty.

The safety margin in the mortality rate described as item (2) above is designed to cover the systematic or non-diversifiable risk of 'mis-estimation' of the expected amounts of life insurance claims. This margin is called the 'provision for adverse deviation' or PAD. This means that the valuation result contains a safety margin that protects, to a certain extent, against the risk that the underlying mortality rates used in the valuation are inadequate.

Diversifiable risk in Canada is provided for by an allocation of surplus under the 'Minimum Continuing Capital and Surplus Requirements' (MCCSR), which use risk-based capital principles.

In this paper, we use Empirical Bayes Credibility Theory to provide a theoretical basis for the calculation of the risk measures associated with mortality risk for insurance companies. Although the diversifiable and nondiversifiable risks are intertwined, we propose a method to separate these risks.

\section{The Available Data}

The data come from the annual studies of individual insurance mortality conducted by the Institute of Insurance and Pension Research at the University of Waterloo for the Canadian Institute of Actuaries. These studies include most but not all individual insurance business in Canada. In total, 19 companies are included in the data covering the period 1984/5$1992 / 3$, with each company contributing data for at least 6 of a possible 9 years. The actual to expected $(\mathrm{A} / \mathrm{E})$ mortality ratios are computed for each year in aggregate for each company. In the annual study, the basis for the expected claims has changed as new mortality tables are adopted. We have adjusted all data to relate to the currently used CIA 86-92 tables, which included separate tables by gender and by smoker/non-smoker status of the insured. 
Due to the confidential nature of the data, we do not give the full data set in this paper. However, we give the results of the analysis of the actual data and the method by which the results have been obtained.

The data is available both by claim numbers and by claim amounts. We give only the results of the credibility analysis by amounts.

\section{Notation AND Assumptions}

Let $S_{i j}$ be a random variable denoting the amount of claims for company $i$ in year $j$, for $i=1,2, \ldots, N, j=1,2, \ldots n_{i}$. For the Canadian data, $N=19$ and $n_{i} \leq 9$; for most companies $n_{i}=9$.

Let $P_{i j}$ denote the expected amount of claims for company $i$ in year $j$, based on standard table mortality, and therefore called the expected tabular claims. This is a measure of the volume of risk, which will be important in allowing for the dependence of the risk on the company size. The expected claims are calculated with reference to the most recent standard table, the CIA 86-92 Table.

Let $X_{i j}=S_{i j} / P_{i j}$ denote the mortality loss ratio (that is, the ratio of actual to expected tabular claims) for company $i$ in year $j$. It is this mortality loss ratio that will be the focus of the analysis.

We make the following assumptions about the random variable $X_{i j}$.

A1. The distribution of $X_{i j}$ depends on some fixed but unknown risk parameter (or vector of parameters), $\theta_{i}$ and on the risk volume $P_{i j}$.

A2. Conditionally for fixed $\theta_{i}$ the random variables $X_{i j} j=1,2, \ldots, n_{i}$ are independent random variables with mean and variance

$$
\mathrm{E}\left[X_{i j} \mid \theta_{i}\right]=\mu\left(\theta_{i}\right) \quad \text { and } \quad \mathrm{V}\left[X_{i j} \mid \theta_{i}\right]=\frac{\sigma^{2}\left(\theta_{i}\right)}{P_{i j}}
$$

and fourth central moment

$$
\mu_{4}\left[X_{i j} \mid \theta_{i}\right]=\frac{3}{P_{i j}^{2}} \sigma^{4}\left(\theta_{i}\right)
$$

for some functions $\mu\left(\theta_{i}\right)$ and $\sigma^{2}\left(\theta_{i}\right)$.

A3. The risk parameters $\theta_{i}$ can be treated as independently and identically distributed random variables drawn from a common distribution.

A4. The pairs $\left(\theta_{i}, X_{i j}\right),\left(\theta_{k}, X_{k l}\right), k \neq i$, are independent random variables.

The first two equations of A2. are the usual assumptions of BühlmannStraub credibility. The second assumption, that $P_{i j} \mathrm{~V}\left[X_{i j} \mid \theta_{i}\right]$ is independent of $j$ is equivalent to assuming that the variance of the aggregate claims given $\theta_{i}$, $V\left[S_{i j} \mid \theta_{i}\right]$ changes in proportion to the risk volume $P_{i j}$. This will only be true if the age and sex profiles of the different portfolios are similar, and if the sums insured are similarly distributed across the different portfolios. If this is not true, a better risk volume would be the square of the standard table expected 
claims divided by the standard table claims variance; that is, if there are $K_{i j}$ contracts in the portfolio of company $i$ in year $j$, with $s_{k}^{i j}$ denoting the sum insured of the $k$ th contract of the portfolio, with appropriate standard table mortality rate $q_{k}^{i j}$, the risk volume would be $V_{i j}$ where:

$$
V_{i j}=\frac{P_{i j}^{2}}{\sum_{k=1}^{K_{i j}}\left(s_{k}^{i j}\right)^{2} q_{k}^{i j}\left(1-q_{k}^{i j}\right)}
$$

The third equation of $\mathrm{A} 2$., for $\mu_{4}\left[X_{i j} \mid \theta_{i}\right]$, is used by Centeno (1989) and follows from assuming that the coefficient of kurtosis of individual risks is equal to 3.0, which is the kurtosis coefficient of the Normal distribution.

The final assumption, A4., implies that the successive year's results for any company are independent of each other except through the risk parameter; and that the results between companies are independent. What is ruled out by this assumption is an overall 'year effect' on mortality to which all companies are subject. In practice, the fact that mortality experience tends to improve year to year constitutes such a 'year effect'. We have therefore adjusted all the data to eliminate the effect of mortality improvements - that is, all data has been adjusted to the mortality levels appropriate to the latest standard tables. A constant adjustment factor was applied to the claims data for each portfolio for each year, calculated such that the overall mortality experience of the whole industry data each year was equal to the standard table expected mortality of that year.

\section{THE 'BEST ESTIMATE' OF THE MORTALITY LOSS RATIO}

In terms of the notation above, the objective is to find the best estimate of $\mathrm{E}\left[X_{i n+1} \mid \theta_{i}\right]=\mu\left(\theta_{i}\right)$, which is unknown, given the set of observed values for company $i, \underline{X}_{i}=X_{i 1}, \ldots, X_{i n_{i}}$, and the assumptions above. We denote this estimate $\tilde{\mu}_{i}$ for company $i, i=1,2, \ldots, N$.

Let $P_{i}=\sum_{j=1}^{n_{i}} P_{i j}$; that is, $P_{i}$ is the total expected claims for company $i$ over the $n_{i}$ years of data;

The Bühlmann-Straub credibility estimator $\tilde{\mu}_{i}$ of $\mu\left(\theta_{i}\right)$ is the estimator which is linear in the data, $X_{i 1}, \ldots, X_{i n_{i}}$, and which minimizes the squared error loss over all such linear estimators, that is, for any linear estimator $\tilde{\mu}\left(\theta_{i}\right)$ :

$$
\mathrm{E}\left[\left(\tilde{\mu}_{i}-\mu\left(\theta_{i}\right)\right)^{2}\right] \leq \mathrm{E}\left[\left(\tilde{\mu}\left(\theta_{i}\right)-\mu\left(\theta_{i}\right)\right)^{2}\right]
$$

The solution to this (which is the best linear estimator of $\mathrm{E}\left[\mu\left(\theta_{i}\right) \mid \underline{X}_{i}\right]$ ), has the form:

$$
\tilde{\mu}_{i}=Z_{i} \bar{X}_{i}+\left(1-Z_{i}\right) \mathrm{E}\left[\mu\left(\theta_{i}\right)\right]
$$


where $Z_{i}$ is the credibility factor for company $i$, and is found from the equation:

$$
Z_{i}=\frac{P_{i}}{P_{i}+\phi} \quad \text { and } \quad \phi=\frac{\mathrm{E}\left[\sigma^{2}\left(\theta_{i}\right)\right]}{\mathrm{V}\left[\mu\left(\theta_{i}\right)\right]}
$$

and where the mean $\bar{X}_{i}$ is the weighted mean of the data for company $i$ weighted by the expected claims, $P_{i j}$; that is, if there are $n_{i}$ years of data,

$$
\bar{X}_{i}=\frac{1}{P_{i}} \sum_{j=1}^{n_{i}} P_{i j} X_{i j}
$$

As the risk parameters, $\left\{\theta_{i}\right\}_{i=1}^{N}$, are assumed to be identically distributed, moments of functions of $\theta_{i}$ are identical for all companies. Hence, $\phi$ and $\mathrm{E}\left[\mu\left(\theta_{i}\right)\right]$ are the same for all companies. We will estimate these quantities from the aggregated industry data.

\section{MORTALITY MARGIN FOR NON-DIVERSIFIABLE RISK}

We can measure the accuracy of the estimator $\tilde{\mu}_{i}$ using the expected quadratic loss,

$$
\mathrm{E}\left[\left(\mu\left(\theta_{i}\right)-\tilde{\mu}_{i}\right)^{2}\right]=\left(1-Z_{i}\right) V\left[\mu\left(\theta_{i}\right)\right]
$$

We have denoted this $\tilde{\nu}_{i}$.

The margin for non-diversifiable risk is expressed as an addition to the best estimate mortality (PAD) to allow for the risk of mis-estimation of the expected deaths.

The margin used depends on the required probability ( $p$, say) that the valuation mortality loss ratio is greater than the true company mortality loss ratio. That is, we want to find a margin $m_{p}(i)$ for company $i$ such that

$$
\operatorname{Pr}\left[\mu\left(\theta_{i}\right)<\tilde{\mu}_{i}+m_{p}(i)\right] \approx p
$$

If we assume that the posterior distribution of $\mu\left(\theta_{i}\right)$ is approximately Normal, then using $\Phi(z)$ to denote the standard Normal distribution function:

$$
\begin{aligned}
& \operatorname{Pr}\left[\mu\left(\theta_{i}\right)<\tilde{\mu}_{i}+\Phi^{-1}(p) \sqrt{\tilde{\nu}_{i}}\right] \approx p \\
& \Rightarrow m_{p}(i)=\Phi^{-1}(p) \sqrt{\tilde{\nu}_{i}}
\end{aligned}
$$




\section{The Fluctuation Reserve}

\subsection{Variance of $X_{i j}$ using credibility}

In the model we are using, the true (but unknown) random variation in the mortality loss ratio for company $i$ in any future year $r$, given expected claims $P_{i r}$, is $\sigma^{2}\left(\theta_{i}\right) / P_{i r}$.

The credibility estimate of $\sigma^{2}\left(\theta_{i}\right)$ is the nearest linear function (in $s_{i}^{2}$ ) to the posterior mean:

$$
\mathrm{E}\left[\sigma^{2}\left(\theta_{i}\right) \mid \underline{X}_{i}\right]=\mathrm{E}\left[P_{i j} V\left[X_{i j} \mid \theta_{i}\right] \mid \underline{X}_{i}\right] .
$$

This estimate has the form, given in Centeno (1989):

$$
\tilde{\sigma}_{i}^{2}=C_{i} s_{i}^{2}+\left(1-C_{i}\right) \mathrm{E}\left[\sigma^{2}\left(\theta_{i}\right)\right]
$$

where

$$
\begin{aligned}
s_{i}^{2} & =\frac{1}{\left(n_{i}-1\right)} \sum_{j=1}^{n_{i}} P_{i j}\left(X_{i j}-\bar{X}_{i}\right)^{2} \\
\text { and } \quad C_{i} & =\frac{1}{1+\frac{2}{n_{i}-1} \varphi} \text { and } \varphi=\frac{\mathrm{E}\left[\sigma^{4}\left(\theta_{i}\right)\right]}{\mathrm{V}\left[\sigma^{2}\left(\theta_{i}\right)\right]}
\end{aligned}
$$

This estimate is an estimate of $P_{i j} \mathrm{E}\left[\mathrm{V}\left[X_{i j} \mid \theta_{i}\right] \mid \underline{X}_{i}\right]$.

All of the factors $\mathrm{E}\left[\sigma^{2}\left(\theta_{i}\right)\right], \mathrm{E}\left[\sigma^{4}\left(\theta_{i}\right)\right], \mathrm{V}\left[\sigma^{2}\left(\theta_{i}\right)\right]$ are independent of the company, as the $\left\{\theta_{i}\right\}$ are assumed identically distributed. Thus, the only company dependent variable in $C_{i}$ is $n_{i}$, that is, the number of years data available for the company.

\subsection{Variance of the Annual Death Strain}

The diversifiable risk is the risk to solvency from random variation in claims experience. In Canada it has been the view that this risk is best covered by an allocation of capital (under the Minimum Continuing Capital and Surplus Requirements, or MCCSR).

This capital requirement is held in addition to the reserve of the company. Hence, the variation that is important is not the variance of total claims, but the variance of the death strain, which is defined as the excess of sums insured over the year-end reserves in respect of the policies becoming claims through death.

Consider the (random) death strain for company $i$ in year $j$, denoted $D_{i j}$. Let $a_{k}^{i j}$ be the net amount at risk (NAAR) for the $k$ th contract of company $i$ in year $j$. The net amount at risk for an individual contract is the sum insured 
minus the year end reserve required if the policyholder survives (also known as the death strain at risk). Let $I_{k}$ be an indicator variable for that contract, where $I_{k}=0$ if the life survives and $I_{k}=1$ if the life dies in year $j$. Then

$$
D_{i j}=\sum_{k} a_{k}^{i j} I_{k}
$$

In order to find the variance of the death strain we need some estimate of $\operatorname{Pr}\left[I_{k}=1\right]$; we assume that, given $X_{i j}$, this probability is given by $q_{k}^{i j} X_{i j}$, where $q_{k}^{i j}$ is the standard table mortality rate for the individual contract.

An estimate of the variability of the death strain is given by

$$
\begin{aligned}
\mathrm{E}\left[\mathrm{V}\left[D_{i j} \mid \theta_{i}\right]\right]=\mathrm{E}\left[\mathrm{E}\left[\mathrm{V}\left[D_{i j} \mid X_{i j}\right] \mid \theta_{i}\right]+\mathrm{V}\left[\mathrm{E}\left[D_{i j} \mid X_{i j}\right] \mid \theta_{i}\right]\right] \\
=\mathrm{E}\left[\mathrm{E}\left[\sum_{k}\left(a_{k}^{i j}\right)^{2} q_{k}^{i j} X_{i j}\left(1-q_{k}^{i j} X_{i j}\right) \mid \theta_{i}\right]+\mathrm{V}\left[\sum_{k} a_{k}^{i j} q_{k}^{i j} X_{i j} \mid \theta_{i}\right]\right] \\
=\mathrm{E}\left[\sum_{k}\left(a_{k}^{i j}\right)^{2} q_{k}^{i j} \mu\left(\theta_{i}\right)-\sum_{k}\left(a_{k}^{i j}\right)^{2}\left(q_{k}^{i j}\right)^{2}\left(\mu\left(\theta_{i}\right)^{2}+\frac{\sigma^{2}\left(\theta_{i}\right)}{P_{i j}}\right)\right. \\
\left.+\left(\sum_{k} a_{k}^{i j} q_{k}^{i j}\right)^{2} \frac{\sigma^{2}\left(\theta_{i}\right)}{P_{i j}}\right]
\end{aligned}
$$

We substitute the credibility estimators for $\mu\left(\theta_{i}\right)$ and $\sigma^{2}\left(\theta_{i}\right)$ to give an estimate of the variance of the death strain of:

$$
\left(\sum_{k}\left(a_{k}^{i j}\right)^{2} q_{k}^{i j}\right) \tilde{\mu}_{i}-\left(\sum_{k}\left(a_{k}^{i j}\right)^{2}\left(q_{k}^{i j}\right)^{2}\right)\left(\frac{\tilde{\sigma}_{i}^{2}}{P_{i j}}+\tilde{\mu}_{i}^{2}\right)+\left(\sum_{k} a_{k}^{i j} q_{k}^{i j}\right)^{2}\left(\frac{\tilde{\sigma}_{i}^{2}}{P_{i j}}\right)
$$

In practice, the middle term is negligible in comparison with the first and third terms.

We may determine an approximate $99.9 \%$ contingency provision of, say, 3.090 times the square root of the estimated variance.

\section{Estimation of the Structural Parameters}

The structural parameters are all those parameters which are common to all companies because of the assumption that $\theta_{i}$ are identically distributed.

We have used the following estimators of the structural parameters, using the aggregate industry data. These are all unbiased, but are not the only

possible meaningful estimators.
The estimate of $\mathrm{E}\left[\mu\left(\theta_{i}\right)\right]$ that we have used is, $\hat{\mu}=\sum_{i=1}^{N} Z_{i} \bar{X}_{i} / \sum_{i=1}^{N} Z_{i}$ 
The estimate of $\mathrm{E}\left[\sigma^{2}\left(\theta_{i}\right)\right]$ which we have used is $\widehat{\sigma_{2}}$, where

$$
\widehat{\sigma_{2}}=\sum_{i=1}^{N} C_{i} s_{i}^{2} / \sum_{i=1}^{N} C_{i}
$$

The estimate of $\mathrm{V}\left[\mu\left(\theta_{i}\right)\right]$ which we have used is $\hat{\nu}$ where $\hat{\nu}=\frac{P W-\widehat{\sigma_{2}}}{\Pi P}$ and where $\Pi=\sum_{i=1}^{N} \frac{P_{i}}{P}\left(1-\frac{P_{i}}{P}\right) /\left\{\left(\sum_{i=1}^{N} n_{i}\right)-1\right\}$ and $W=\sum_{i=1}^{N} \sum_{j=1}^{n_{i}} \frac{P_{i j}}{P}\left(X_{i j}-\hat{\mu}\right)^{2} /\left\{\left(\sum_{i=1}^{N} n_{i}\right)-1\right\}$

The estimate of $\mathrm{E}\left[\sigma^{4}\left(\theta_{i}\right)\right]$ is $\widehat{\sigma}_{4}$ say, where

$$
\widehat{\sigma_{4}}=\left(\frac{\sum_{i=1}^{N}\left(n_{i}-1\right)\left(s_{i}^{2}\right)^{2}}{\sum_{i=1}^{N}\left(n_{i}+1\right)}\right)
$$

and the estimate of $\mathrm{V}\left[\sigma^{2}\left(\theta_{i}\right)\right]$ is $\widehat{{\sigma^{2}}^{2}}$ say, where

$$
\widehat{\nu_{\sigma^{2}}}=\left\{\left(\sum_{i=1}^{N}\left(n_{i}-1\right)\left(s_{i}^{2}-\widehat{\sigma^{2}}\right)^{2}\right)-2 \widehat{\sigma_{4}}(N-1)\right\} / R
$$

where

$$
R=\sum_{i=1}^{N}\left(n_{i}-1\right)-\frac{\sum_{i=1}^{N}\left(n_{i}-1\right)^{2}}{\sum_{i=1}^{N}\left(n_{i}-1\right)}
$$

In practice all of these parameters could be estimated by a central data collecting organisation. Individual companies could then be told of the results to use in their own calculations.

\section{NUMERICAL RESULTS OF THE INDUSTRY AND COMPARISON WITH CURRENT STANDARDS}

\subsection{Valuation mortality: 'best estimate' and margins}

In Table 1 the 'best estimate' credibility calculations are shown for some of the companies who contribute to the Canadian mortality study.

The credibility estimate of the expected mortality loss ratio, $\mu_{i}$ is given in column (3). 
The level of safety margin deemed appropriate is an subjective choice reflecting the perceived need for security. In Canada, the valuation assumptions are selected each year which suggests that there are opportunities to adjust each year. Also, a less stringent standard would be appropriate for participating business compared with non-participating because of the opportunity to adjust for excess mortality by adjusting dividends.

Column (5) of Table 1 gives the safety margin for mortality required at a safety level of $90 \%$; this is just 1.28 times the standard error given in column (4). Column (6) gives the total mortality loss ratio to be used, as a multiple of the standard table. This is the sum of the best estimate and the $90 \%$ margin.

TABLE 1

RESULTS OF CREDIBILITY ANALYSIS OF REAL INDUSTRY DATA; BEST ESTIMATE AND MISESTIMATION MARGIN FOR ACTUAL/EXPECTED MORTALITY

\begin{tabular}{cccccc}
\hline \hline $\begin{array}{c}\text { Company } \\
\text { number } i\end{array}$ & Cred. Factor $Z_{i} \begin{array}{c}\text { Cred. Estimate } \\
\text { of } A / S T, \tilde{\mu}_{i}\end{array}$ & $\begin{array}{c}\text { St. Error } \\
\text { of } \tilde{\mu}_{i}, \sqrt{\tilde{\nu}_{i}}\end{array}$ & $\begin{array}{c}90 \% \text { margin } \\
1.28 \sqrt{\tilde{\nu}_{i}}\end{array}$ & $\tilde{\mu}_{i}+1.28 \sqrt{\tilde{\nu}_{i}}$ \\
$(1)$ & $(2)$ & $(3)$ & $(4)$ & $(5)$ & $(6)$ \\
\hline 1 & 0.80 & 0.796 & 0.051 & 0.065 & 0.861 \\
2 & 0.70 & 1.024 & 0.061 & 0.079 & 1.103 \\
3 & 0.70 & 0.916 & 0.061 & 0.078 & 0.994 \\
4 & 0.84 & 0.983 & 0.046 & 0.058 & 1.041 \\
5 & 0.94 & 1.099 & 0.028 & 0.036 & 1.135 \\
6 & 0.86 & 0.919 & 0.043 & 0.055 & 0.974 \\
7 & 0.80 & 1.143 & 0.051 & 0.065 & 1.208 \\
8 & 0.92 & 0.912 & 0.032 & 0.041 & 0.953 \\
9 & 0.70 & 1.120 & 0.062 & 0.079 & 1.199 \\
10 & 0.83 & 1.052 & 0.046 & 0.060 & 1.112 \\
11 & 0.87 & 0.906 & 0.040 & 0.051 & 0.957 \\
12 & 0.91 & 0.943 & 0.033 & 0.042 & 0.985 \\
13 & 0.29 & 1.049 & 0.095 & 0.121 & 1.170 \\
14 & 0.83 & 1.179 & 0.046 & 0.059 & 1.238 \\
15 & 0.70 & 1.092 & 0.062 & 0.079 & 1.171 \\
16 & 0.72 & 0.943 & 0.060 & 0.076 & 1.019 \\
17 & 0.41 & 0.985 & 0.086 & 0.110 & 1.095 \\
18 & 0.80 & 1.047 & 0.051 & 0.065 & 1.112 \\
19 & 0.42 & 0.978 & 0.086 & 0.110 & 1.088 \\
\hline \hline
\end{tabular}


The 'best estimate' mortality ranges from around $80 \%$ to $118 \%$ of the standard table mortality. The valuation basis mortality margin broadly has the role of the current 'provision for adverse deviation' in the Canadian regulations. The values here range from around $4 \%$ to around $12 \%$ using a $90 \%$ safety margin.

A direct comparison with the current Canadian PAD levels is difficult as the current PADs are age dependent; they are set at some level between $0.015 / e_{x}$ and $0.00375 / e_{x}$. For an average policyholder $e_{x}$ might be approximately 30 , giving an addition of between 0.0005 and 0.000125 to the mortality rate. This is difficult to compare with the proposed provision as they are very sensitive to age - at early ages an addition of 0.0005 might be a $50 \%$ loading on the mortality rate; at later ages it would be around $2 \%$. The margins derived here, using a $90 \%$ standard do not appear significantly out of line with current provisions.

\subsection{The surplus needs (or fluctuation reserve); comparison with current standards}

Given the estimated standard deviation of the death strain, from Section 6.2, we may determine an approximate $99.9 \%$ contingency provision of, say, 3.090 times the estimated standard deviation of death strain.

An accurate assessment of $s d\left[D_{i j}\right]$ requires individual policy information, which is unavailable for this study. In order to compare very roughly the proposed standard with the current standard, we have made some sweeping approximations.

1. First, we assume a constant standard table mortality rate for all business of $q_{k}^{i j}=0.0017$.

2. We also assume that

$$
\sum_{k}\left(a_{k}^{i j}\right)^{2} \approx \frac{4}{K_{i j}}\left(\sum_{k} a_{k}^{i j}\right)^{2}
$$

where $K_{i j}$ is the number of contracts of company $i$ in year $j$. The factor 4 is the average of the squared NAARs divided by the square of the average of the NAARs, and was estimated for this study by looking at the sum assured data of one large portfolio. The factor 4 is appropriate if the distribution of the net amount at risk (NAAR) over the policies is, say, Pareto, (also known as American Pareto) with shape parameter $\alpha=3-$ that is, taking a random policy, the probability that the NAAR is less than some amount $A$ say, is $1-(\lambda /(\lambda+A))^{\alpha}$. The Pareto distribution has been used elsewhere for sums assured - for example, by Pentikäinen and Pesonen (1988), who used a truncated version, with shape parameter $\alpha=2.5$. As much of the business is term assurance, where the reserve is a fairly level proportion of the sum assured, the sum assured distribution should be very similar (suitably rescaled) to the NAAR distribution. The 
factor of 4 appears to be appropriate for the Canadian data, and is consistent (allowing for truncation) with the Pentikäinen and Pesonen distribution, but may not be representative of other portfolios. For endowment assurances, we expect the sum assured distribution to have a lower coefficient of variance, but the effect of increasing reserves would be to give a NAAR distribution with, perhaps, a higher coefficient of variance (and lower mean) than that of a predominantly term assurance portfolio.

2a.In fact the results are not very sensitive to this assumption. We also give (in parentheses) the results adopting an alternative assumption that

$$
\sum_{k}\left(a_{k}^{i j}\right)^{2} \approx \frac{6}{K_{i j}}\left(\sum_{k} a_{k}^{i j}\right)^{2}
$$

that is, assuming a factor of 6 for $\sum_{k}\left(a_{k}^{i j}\right)^{2} /\left(\left(\sum_{k} a_{k}^{i j}\right)^{2} / K_{i j}\right)$, in place of 4 .

3. Based on the analysis of the company data, the results of which are summarised in Section 8.3, we assume that for a small portfolio of, say, 50,000 contracts, the value of $\frac{\tilde{\sigma}_{i}^{2}}{P_{i j}}$ is approximately 0.15 . The value for a medium portfolio of, say, 200,000 policies is assumed to be approximately 0.03 and a value of 0.01 is used for a large portfolio of, say, $1,000,000$ policies.

4. Finally, we assume that $\tilde{\mu}_{i} \approx 1.0$.

Based on these approximations, we estimate the $99.9 \%$ fluctuation reserves per unit of NAAR, as

$$
3.090 \sqrt{\left(q \frac{4}{K}+q^{2}\left(\frac{\tilde{\sigma}_{i}^{2}}{P_{i j}}+\tilde{\nu}_{i}\right)\right)}
$$

where $q$ is 0.0017 and $\mathrm{K}$ is the number of policies. This gives the following estimated fluctuation requirements:

- Small company $(50,000$ policies $)$

The reserve is approximately $0.23 \%$ of the total NAAR (or $0.25 \%$ using assumption 2a. above). This compares with a current requirement of $0.25 \%$ of the total NAAR for small non-profit portfolios.

- Medium company (200,000 policies)

The reserve is approximately $0.11 \%$ of the total NAAR (or $0.12 \%$ using assumption 2a). This compares with a current requirement for a nonprofit portfolio of around $0.2 \%$ of the total NAAR.

- Large company $(1,000,000$ policies $)$

The reserve is approximately $0.06 \%$ of the total NAAR (also $0.06 \%$ using assumption 2a). This compares with a current requirement for a non-profit portfolio of around $0.12 \%$ of the total NAAR. 
The requirements based on these assumptions appear to be less onerous for medium and large portfolios than the current requirements. Current requirements for with-profit portfolios are one-half of the non-profit requirements.

\subsection{Approximate fluctuation reserves using industry data}

We have used

$$
P_{i j}^{2}\left(\frac{\tilde{\sigma}_{i}^{2}}{P_{i j}}\right)=\left(\sum_{k} S_{k}^{i j} q_{k}^{i j}\right) \tilde{\sigma}_{i}^{2}
$$

as a simple approximation to the variance of the death strain, where $S_{k}^{i j}$ is the individual contract sum assured for the $k$ th contract of company $i$ in year $j$. That is, we assumed that using the sum assured in place of the death strain in the final term would roughly compensate for omitting the first two terms. This is reasonably accurate using the Pareto assumption from 8.2 above, and assuming further that the NAAR is approximately $90 \%-95 \%$ of the sum assured. The majority of life insurance written in Canada is term assurance; for these contracts the reserve is commonly small in proportion to the sum insured. It should be emphasised that this approximation is for illustration only, in practice the true company NAAR should be used. This allows high margins in the reserving to offset the fluctuation reserve requirement.

The results are given in Table 2. The reserving standard is $99.9 \%$, which means that the surplus requirement is calculated to be sufficient to cover claims fluctuation with probability of approximately 0.999 . The reserve is shown per $\$ 100$ dollars expected claims, for a medium sized portfolio, with $\$ 20$ million expected claims. The larger portfolios are perhaps two to four times bigger; the approximated reserve per cent of expected claims would then be 1.5-2 time smaller than those shown here, as the reserve is proportional to the square root of the expected claims. The smallest portfolios would be around four times smaller than the example used; the reserve per cent of expected claims would be around twice those given above. 
TABLE 2

APPROXIMATE SURPLUS REQUIREMENT PER \$100 EXPECTED CLAIMS

\begin{tabular}{ccc}
\hline Company number & $\begin{array}{c}\text { Estimated St. Deviation }(\%) \\
\tilde{\sigma}_{i} / 100\end{array}$ & $\begin{array}{c}\text { Approx } 99.9 \% \text { reserve per } \$ 100 \\
\text { expected claims } P_{i j}=20 \text { million } \\
(1)\end{array}$ \\
\hline 1 & 650.5 & $(3)$ \\
2 & 488.0 & 44.95 \\
3 & 508.7 & 33.71 \\
4 & 559.5 & 35.15 \\
5 & 575.8 & 38.66 \\
6 & 702.6 & 39.78 \\
7 & 488.4 & 48.55 \\
8 & 655.4 & 33.75 \\
9 & $1,390.9$ & 45.29 \\
10 & 440.8 & 96.11 \\
11 & 656.9 & 30.46 \\
12 & 463.6 & 45.43 \\
13 & 776.4 & 32.03 \\
14 & 546.7 & 53.65 \\
15 & 492.2 & 37.78 \\
16 & 698.1 & 34.01 \\
17 & 398.8 & 48.23 \\
18 & 493.1 & 27.56 \\
19 & 502.4 & 34.07 \\
\hline
\end{tabular}

\section{CONCLUSIONS}

A number of simplifying assumptions were made in order to obtain tractable results. As a result the numbers should be viewed as approximations. Since some subjectivity is required in any case, because arbitrary safety margins are selected, the loss in information is somewhat mitigated. A few areas should be identified.

First, the variance assumption of A2. Section 3 is only approximately correct due to different policy sizes and different age/sex profiles. Secondly, due to data restrictions, we are not able to identify any reinsurance. Reinsurance reduces risk exposure. Our calculations were gross of reinsurance ceded. Consequently, our results will be conservative.

Finally, the practice in Canada has been to consider the provision for adverse deviations as protection against systematic deterioration in experience on the future. Our methodologies assume that there are no trends. To accommodate that assumption, we detrended the data to eliminate the downward trend. As a result, our results are conservative in 
the sense that they assume no future improvements in mortality, but they are not sufficiently conservative for a hypothetical increase in future mortality levels.

\section{ACKNOWLEDGEMENTS}

The authors thank Julia Wirch of the University of Waterloo for research assistance, and Lourdes Centeno and Howard Waters for constructive comments. The two anonymous referees are also thanked for helpful improvements to the paper.

\section{REFERENCES}

Bühlmann, H. and STraub, E. (1970). Glaubwürdigkeit für Schadensätze. Bulletin of the Association of Swiss Actuaries 70, 111-133.

Centeno, M.L. (1989). The Bühlmann-Straub model with the premium calculated according to the variance principle. Insurance: Mathematics and Economics 8, 3-10.

HARdY, M.R. and PANJER, H.H. (1996). Mortality Risk: Margin and Capital Needs. Institute of Insurance and Pensions Research, University of Waterloo. Research Report 96-10.

Klugman, S., Panjer, H.H. and Willmot, G.E. (1997). Loss Models: From Data to Decisions. John Wiley and Sons (forthcoming).

Pentikäinen, T. and Pesonen, M. (1988). Stochastic dynamic analysis of life insurance. Transactions of the 23rd International Congress of Actuaries 1, p. 421.

\section{APPENDIX}

\section{UNBIASEDNESS OF STRUCTURAL PARAMETER ESTIMATORS}

All of the estimators are unbiased; $\hat{\nu}$ is derived in Klugman et al (1997). For $\widehat{\sigma_{4}}$ we use the result from Centeno (1989) that

$$
\begin{aligned}
& V\left[s_{i}^{2}\right]=\mathrm{V}\left[\sigma^{2}\left(\theta_{i}\right)\right]+\frac{2}{n_{i}-1} \mathrm{E}\left[\sigma^{4}\left(\theta_{i}\right)\right] \\
& \Rightarrow \mathrm{E}\left[s_{i}^{4}\right]=\mathrm{E}\left[s_{i}^{2}\right]^{2}+\mathrm{V}\left[\sigma^{2}\left(\theta_{i}\right)\right]+\frac{2}{n_{i}-1} \mathrm{E}\left[\sigma^{4}\left(\theta_{i}\right)\right] \\
& \text { but } \mathrm{E}\left[s_{i}^{2}\right]=\mathrm{E}\left[\sigma^{2}\left(\theta_{i}\right)\right] \\
& \text { so } \mathrm{E}\left[s_{i}^{4}\right]=\mathrm{E}\left[\sigma^{4}\left(\theta_{i}\right)\right]\left(1+\frac{2}{n_{i}-1}\right) \\
& \Rightarrow\left(n_{i}-1\right) \mathrm{E}\left[s_{i}^{4}\right]=\left(n_{i}+1\right) \mathrm{E}\left[\sigma^{4}\left(\theta_{i}\right)\right] \\
& \Rightarrow \mathrm{E}\left[\sum_{i=1}^{N}\left(n_{i}-1\right) s_{i}^{4}\right]=\sum_{i=1}^{N}\left(n_{i}+1\right) \mathrm{E}\left[\sigma^{4}\left(\theta_{i}\right)\right] \\
& \Rightarrow \widehat{\sigma_{4}}=\left(\frac{\sum_{i=1}^{N}\left(n_{i}-1\right)\left(s_{i}^{2}\right)^{2}}{\sum_{i=1}^{N}\left(n_{i}+1\right)}\right)
\end{aligned}
$$


For $\widehat{\nu_{\sigma^{2}}}$ we consider

$$
\begin{aligned}
& \xi=\sum_{i=1}^{N}\left(n_{i}-1\right)\left(s_{i}^{2}-\widehat{\sigma}_{2}\right)^{2} \\
& \text { let } M=\sum_{i=1}^{N}\left(n_{i}-1\right) \quad \text { so } \quad \widehat{\sigma}_{2}=\frac{\sum_{i=1}^{N}\left(n_{i}-1\right) s_{i}^{2}}{M} \\
& \mathrm{E}[\xi]=\sum_{i=1}^{N}\left(n_{i}-1\right) \mathrm{E}\left[s_{i}^{4}\right]-2 \mathrm{E}\left[\sum_{i=1}^{N}\left(n_{i}-1\right) s_{i}^{2} \widehat{\sigma_{2}}\right]+\mathrm{E}\left[\widehat{\sigma}_{2}^{2}\right] M \\
& \sum_{i=1}^{N}\left(n_{i}-1\right) \mathrm{E}\left[s_{i}^{4}\right]=\sum_{i=1}^{N}\left(n_{i}-1\right) \mathrm{V}\left[s_{i}^{2}\right]+M \mathrm{E}\left[\sigma^{2}\left(\theta_{i}\right)\right]^{2} \\
& \text { and } \mathrm{E}\left[\sum_{i=1}^{N}\left(n_{i}-1\right) s_{i}^{2} \widehat{\sigma}_{2}\right]=\sum_{i}\left(n_{i}-1\right)^{2} \frac{V\left[s_{i}^{2}\right]}{M}+M \mathrm{E}\left[\sigma^{2}\left(\theta_{i}\right)\right]^{2} \\
& \text { and } \mathrm{E}\left[\widehat{\sigma}_{2}^{2}\right] M=\sum_{i=1}^{N}\left(n_{i}-1\right)^{2} \frac{V\left[s_{i}^{2}\right]}{M}+M \mathrm{E}\left[\sigma^{2}\left(\theta_{i}\right)\right]^{2} \\
& \text { so } \mathrm{E}[\xi]=\sum_{i=1}^{N} \mathrm{~V}\left[s_{i}^{2}\right]\left(\left(n_{i}-1\right)-\frac{\left(n_{i}-1\right)^{2}}{M}\right)
\end{aligned}
$$

Substitute $\mathrm{V}\left[\sigma^{2}\left(\theta_{i}\right)\right]+\frac{2}{n_{i}-1} \mathrm{E}\left[\sigma^{4}\left(\theta_{i}\right)\right]$ for $\mathrm{V}\left[s_{i}^{2}\right]$

$$
\mathrm{E}[\xi]=\mathrm{V}\left[\sigma^{2}\left(\theta_{i}\right)\right] \sum_{i=1}^{N}\left(\left(n_{i}-1\right)-\frac{\left(n_{i}-1\right)^{2}}{M}\right)+2 \mathrm{E}\left[\sigma^{4}\left(\theta_{i}\right)\right](N-1)
$$

that is $\mathrm{E}[\xi]=\mathrm{V}\left[\sigma^{2}\left(\theta_{i}\right)\right] R+\mathrm{E}\left[\sigma^{4}\left(\theta_{i}\right)\right] 2(N-1)$

Hence an unbiased estimator for $\mathrm{V}\left[\sigma^{2}\left(\theta_{i}\right)\right]$, given that $\widehat{\sigma_{4}}$ is an unbiased estimator of $\mathrm{E}\left[\sigma^{4}\left(\theta_{i}\right)\right]$, is

$$
\frac{\xi-\widehat{\sigma_{4}} \cdot(2(N-1))}{R}
$$

\section{MARY Hardy}

Department of Statistics and Actuarial Science University of Waterloo

Waterloo, Ontario

Canada N2L $3 G 1$ 